\title{
Observing programmes of the Observatorio Astronómico Los Molinos, Uruguay
}

\author{
Gonzalo Tancredi ${ }^{1,2}$, S. Roland ${ }^{1}$, R. Salvo ${ }^{1}$, F. Benitez ${ }^{1,2}$, \\ S. Bruzzone ${ }^{1,2}$, A. Ceretta $^{1}$, E. Acosta ${ }^{1}$ \\ ${ }^{1}$ Department of Astronomy, Universidad de la República \\ Iguá 4225, 11400 Montevideo, Uruguay \\ email: gonzalo@fisica.edu.uy \\ ${ }^{2}$ Observatorio Astronómico Los Molinos \\ Cno. de Los Molinos 5769 y Cno. Uruguay, 12400 Montevideo, Uruguay \\ email: oalm@fisica.edu.uy
}

\begin{abstract}
The Observatorio Astronómico Los Molinos (OALM) is the only professional observatory in Uruguay. Its research activity is dedicated to the study of Small Bodies of the Solar System, i.e. comets and asteroids. It is one of the few observatories in the southern hemisphere dedicated to this topic. The observational programmes that we are conducting at present are described, putting especial emphasis in the results obtained last year.
\end{abstract}

Keywords. Astronomy in Uruguay, Observatorio Los Molinos, comets and asteroids

\section{Introduction}

The Observatorio Astronómico Los Molinos (OALM) was inaugurated in 1993 and belongs to the Innovation, Sciences and Technology Office (DICYT) of the Culture and Education Ministry (MEC). It is supported by the Department of Astronomy of Universidad de la República. It is the only professional observatory in Uruguay. It is located in the northern border of Montevideo, the state capital. Therefore, the light pollution issues are worrisome. The latter fact lead us to build another observing facility placed in the country side, in the Province of Maldonado (250 km from Montevideo).

The scientific activity of OALM is focused on Solar System Minor Bodies (i.e. comets and asteroids), being one of the few observatories dedicated to this topic in the southern hemisphere (see Fig. 1 with the distribution of the observatories that have contributed more than 100 astrometric reports to the Minor Planet Center in 2005). The main instruments placed in the OALM' facilities are CCD-equipped 35 and $46 \mathrm{~cm}$ telescopes used for astrometrical and photometrical programmes. The acquisition of the latter instrument, Centurion 18 (Astroworks) give birth to the research programme called Búsqueda Uruguaya y Seguimiento de Cometas y Asteroides - BUSCA. Also in the OALM facilities are placed other observing instruments with their respective domes that belong to amateur astronomers. A detail information about our facilities is found in our institutional website: http://oalm.astronomia.edu.uy

In the framework of the BUSCA programme, the following activities are developed in the observatory:

- Follow-up and confirmation of Near-Earth Objects (NEOs).

- Search for NEOs in the direction of their orbits radiant.

- Photometric follow-up of comets.

- Photometric profiles of asteroids in cometary orbits (ACOs). 


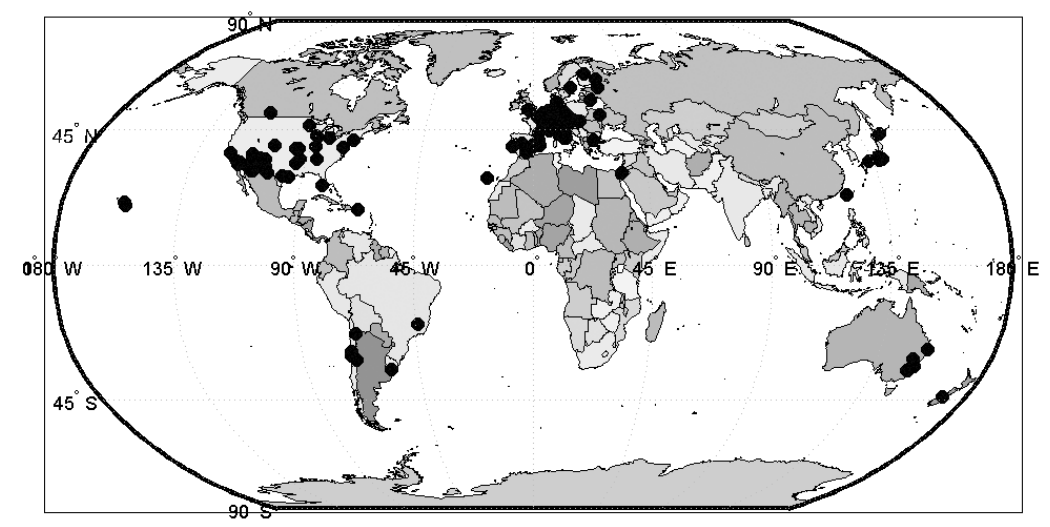

Figure 1. Distribution of observatories that have contributed more than 100 astrometric reports to the Minor Planet Center in 2005. There are 138 observatories in the northern hemisphere, and 15 in the south. OALM is ranked \#7 in asteroids reports and \#4 in comets reports in the southern hemisphere.

In the following sections we describe in detail the previous observing programmes and present some results.

\section{Follow-up and confirmation of Near-Earth Objects (NEOs)}

Through the astrometric reports done in OALM we contribute to the improvement in the determination of the orbital elements of NEOs and the estimate of their impact risk. We observe recently discovered asteroids (i.e. the confirmation task), or already discovered ones that need further observations (i.e. the astrometric follow-up). The confirmation process briefly consists in checking the object list in the NEO Confirmation Page (NEOCP) of the Minor Planet Center (MPC) every night. The object is then observed in the predicted ephemeris and the astrometry is done and reported back to the MPC. An inspection of the physical appearance is done in order to find possible cometary comae. In such a case, the object should be catalogued as a comet. An example of this event was the confirmation of comet C/2006 A1 (Pojmanski) accomplished in January 2006 in OALM (IAUC 8653). (see Fig. 2).

In Fig. 3 the evolution of astrometric reports in the period $1997-2005$ is presented (a) and a distribution among the months for year $2005(b)$. We notice the increment of reports during year 2005 as a result of the massive usage of the 18 inch instrument (Centurion) for this job. From the monthly distribution of reports, we conclude that the winter season is the less productive epoch in the entire year due to bad atmospheric conditions. Unfortunately this is the epoch in which the ecliptic reaches the highest altitude for our sky.

In Fig. 4 the declination $(a)$ and the observed magnitude $(b)$ distributions are shown. There is a concentration of reports in the southern declinations because of our selection criteria applied on the observed objects. This criteria is based on the observatory shortage in the southern hemisphere which causes the southern celestial hemisphere to be poorly observed. The most frequent magnitude range is $R \sim 17-18$, but seldom reaching magnitudes close to 19 . 


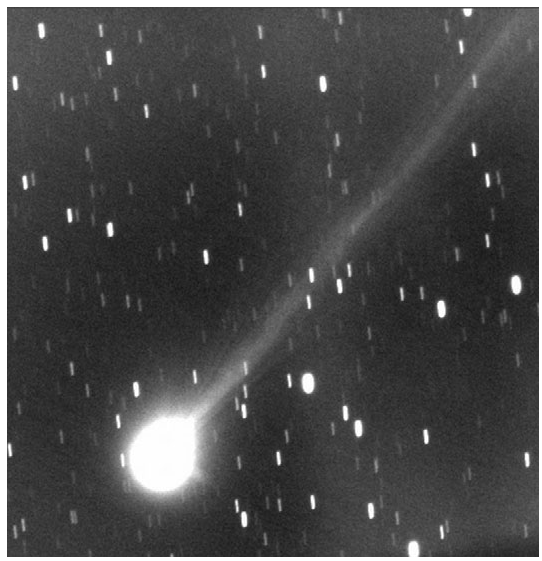

Figure 2. Image of C/2006 A1(Pojmanski) taken at OALM.

a)



Figure 3. Astrometric Reports submitted to the MPC in the period $1997-2005(a)$ and by month during year $2005(b)$. a)

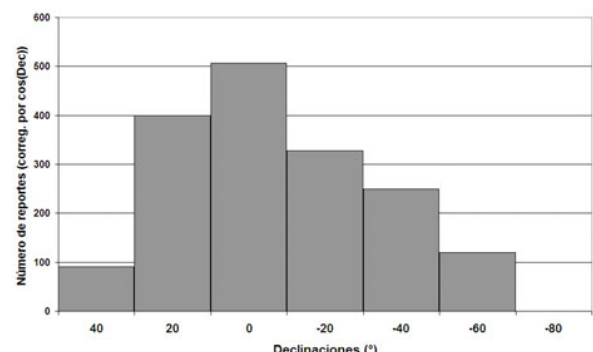

b)



Figure 4. Histogram of the distribution of astrometric reports by declination $(a)$ and by apparent magnitude $(b)$.

\section{Search for NEOs in the direction of their orbits (radiant)}

From the study of the direction of approach of Near-Earth Asteroids (NEAs) to the Earth, we found well-delimited zones in a celestial map where these objects could be observed when they are in direction of collision with the planet. We refer to these positions as the radiants of the orbits, in correspondence with the terminology used in meteor science. If we plot the radiants in a sky map in ecliptic coordinates referred to Sun, the radiants are concentrated in two zones: one close to the anti-solar point and another one centered in the Sun. The anti-solar region is commonly explored by the large survey programmes (i.e: Linear). The regions near the Sun, at low ecliptic latitudes, are filled 
with objects with elongations lower than $30^{\circ}$, making the observations impossible. Elongations suitable for night observations are obtained only for radiants with high ecliptic latitudes.

Since beginning 2006 our survey programme has been concentrated in this region with ecliptic longitudes close to the Sun and high ecliptic latitudes. Due to the annual motion of the Sun, this zone will change their equatorial coordinates giving different configurations during the year. In some months, the densest zones would be in the evening or morning twilight. We generate alt-azimuthal maps of the visible sky at the moment of twilight for each month, centered on the zenith and viewed from inside the celestial sphere. In Fig. 5 evening and morning diagrams are shown for the beginning of each season. The densest and highest region are then selected to point the telescope for NEA searching.
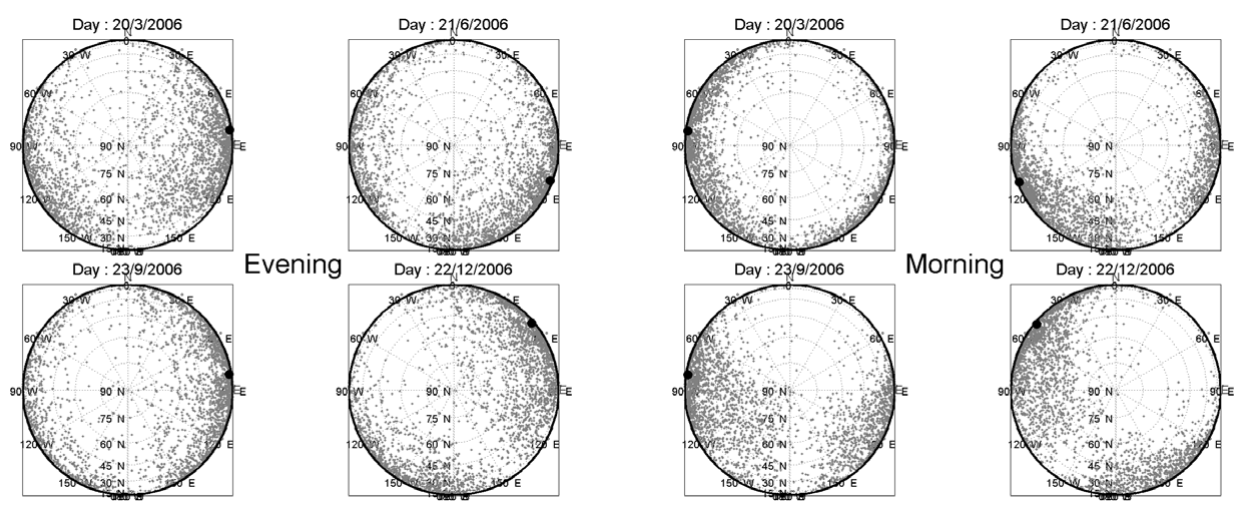

Figure 5. The distribution of NEAs' radiant projected on horizontal coordinates for different epochs of the year, half an hour after (before) sunset (sunrise). The region to look is the highest concentrations of small dots opposite to the large black dot (the anti-Sun).

\section{Photometric follow-up of comets}

The number of astrometric reports of comets between 1997 and 2005 followed the same increase trend as shown in Fig. $3 a$ for NEOs. Concerning comets, astrometric reports are very important for the continuous improvements of the orbital elements and the determination of non-gravitational forces. The non-gravitational parameter can be directly associated with the comet gaseous production, which is obtained from the perihelia light curves (see below), to estimate the mass of the comet( Rickman et al. 1987).

\subsection{Photometry - Perihelion light curve}

We are observing every comet passing through perihelion whenever the magnitude is brighter than $R \sim 18$. In addition to study the action of non-gravitational forces, the light curves are useful to monitor any abrupt magnitude change like outbursts, in order to understand the underlying physical processes like fragmentation (splittings) or the exothermic phase transition of crystalline to amorphous ice.

The photometry of an extended object like the cometary comae is done in the following way: first the astrometric report is done with the software Astrometrica $\dagger$. Second, the photometry is done with the software Focas $\ddagger$. The latter uses the multi-box photometry technique. The photometry is done at several boxes of fixed angular size centered on

$\dagger$ http://www.astrometrica.at/

$\ddagger$ http://www.astrosurf.com/cometas-obs/_Articulos/Focas/Focas.htm 
the comet coordinates. The sizes of these boxes are 10"x10", 20 " $\mathrm{x} 20$ ", 30 " x30", 40" x40" (Fig. 6).
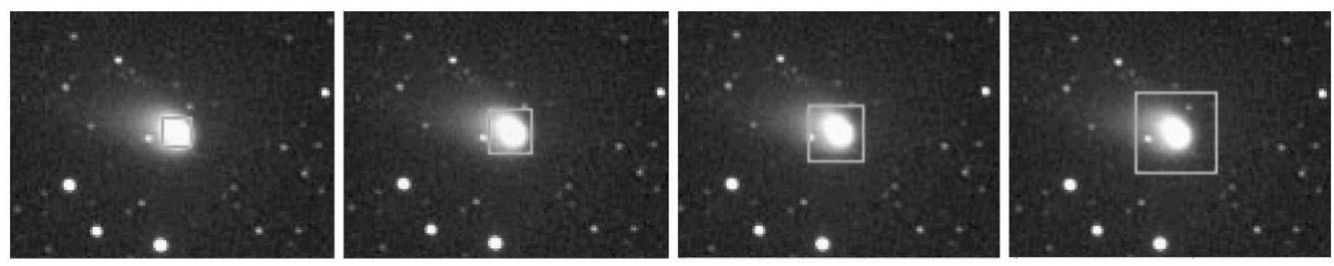

Figure 6. Fixed angular size boxes are shown centered on the comet. Starting from the left, the box sizes are: 10 "x10", 20"x20", 30"x30", 40"x40".

In Fig. 7 the perihelion light curve of comet $37 P /$ Forbes is shown. It was obtained using the OALM's telescopes during year 2005.

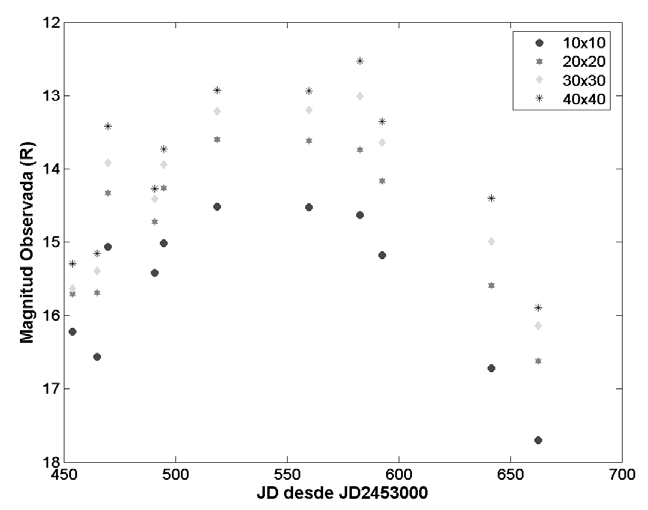

Figure 7. Perihelion light curve for comet $37 P /$ Forbes

Light curves obtained with our small telescopes will allow us to anticipate when the comet will be inactive. We then plan to use larger telescopes to observe and estimate the nuclear magnitude of the comet, as a continuity of the observational programme and the compilation work of cometary nuclear magnitudes that we have been conducting (Licandro et al. 2000, Tancredi et al. 2006).

\section{Photometric profiles of asteroids in cometary orbits (ACOs)}

Physical and dynamical evolution models of comets suggest the possible total deactivation of the gas and dust emission. This occurs by the formation of a dust crust that quenches the gas sublimation. There are some comets with very low levels of activity (Tancredi et al. 2006), a fact that support the existence of dormant comets. Analyzing the distribution of orbital elements for both comets and asteroids, a dynamical criteria was designed in order to classify objects between these two populations. Roughly hundred asteroids were found in cometary orbits and they require a detailed photometric study in order to determine signs of very low activity or a dormant phase.

The photometric follow-up of asteroids in cometary orbits consists in making a profile analysis of the asteroid and compare it to stellar profile in the field, in order to look for possible gaseous activity. This is viewed in the CCD image as an enhancement of the cometary profile over the stellar one, due to the coma. 
The night of $01 / 30 / 2006$, the asteroid 2001 ME1 in a cometary orbit was observed at an heliocentric distance of $1.2 \mathrm{AU}$. The preliminary comparison of the asteroid and the stellar profiles reveals no evident cometary activity. (Fig. 8).
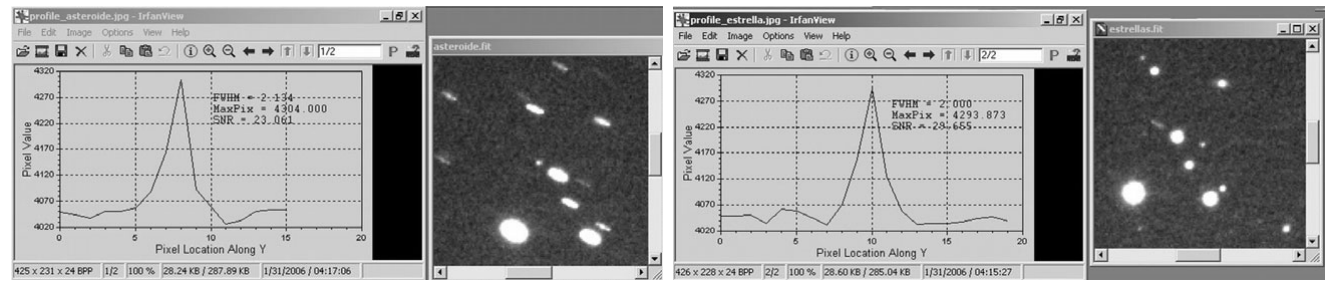

Figure 8. Profile comparison of asteroid 2001 ME1 (left) and a field star (right).

In addition to the photometric studies of comets and asteroids in cometary orbits, we perform the astrometric measurements and report them to the MPC.

\section{Conclusions}

We have presented the research activities that we are conducting at the OALM with relatively small telescopes $(46 \mathrm{~cm}$ and $35 \mathrm{~cm}$ ). We have shown that using these small telescopes for common-day follow-up programmes allows us to make relevant contributions in some scientific niches that are less explored. These small telescopes with easy access are useful for back-up programmes for other more ambitious projects.

We have shown that there are regions in the sky not explored by the big surveys that could be searched for with other small telescopes.

\section{Acknowledgements}

OALM is supported by the contributions of the Education and Culture Ministry, and the Sciences Faculty of Universidad de la República and the Programme for the Development of Basic Sciences (PEDECIBA).

\section{References}

Licandro, J.; Tancredi, G.; Lindgren, M.; Rickman, H.; Gil Hutton, R. 2000, Icarus 147, 161 Rickman, H.; Kamel, L.; Festou, M. C.; Froeschle, Cl. 1987, in ESA, Proc. of the Int. Symposium on the Diversity and Similarity of Comets, p 471

Tancredi, G.; Fern'andez, J.A.; Rickman, H.; Licandro, J. 2006, Icarus 182, 527 